\title{
Characterization of multinucleated giant cells in synovium and subchondral bone in knee osteoarthritis and rheumatoid arthritis
}

\author{
Iván Prieto-Potin ${ }^{1}$, Raquel Largo ${ }^{1}$, Jorge A Roman-Blas ${ }^{1}$, Gabriel Herrero-Beaumont ${ }^{1}$ and David A Walsh ${ }^{2 *}$
}

\begin{abstract}
Background: Multinucleated giant cells have been noticed in diverse arthritic conditions since their first description in rheumatoid synovium. However, their role in the pathogenesis of osteoarthritis (OA) or rheumatoid arthritis (RA) still remains broadly unknown. We aimed to study the presence and characteristics of multinucleated giant cells (MGC) both in synovium and in subchondral bone tissues of patients with OA or RA.
\end{abstract}

Methods: Knee synovial and subchondral bone samples were from age-matched patients undergoing total joint replacement for OA or RA, or non-arthritic post mortem (PM) controls. OA synovium was stratified by histological inflammation grade using index tissue sections. Synovitis was assessed by Krenn score. Histological studies employed specific antibodies against macrophage markers or cathepsin K, or TRAP enzymatic assay.

Results: Inflamed OA and RA synovia displayed more multinucleated giant cells than did non-inflamed OA and PM synovia. There was a significant association between MGC numbers and synovitis severity. A TRAP negative/ cathepsin K negative Langhans-like subtype was predominant in OA, whereas both Langhans-like and TRAP-positive/ cathepsin K-negative foreign-body-like subtypes were most commonly detected in RA. Plasma-like and foam-like subtypes also were observed in OA and RA synovia, and the latter was found surrounding adipocytes. TRAP positive/ cathepsin K positive osteoclasts were only identified adjacent to subchondral bone surfaces. TRAP positive osteoclasts were significantly increased in subchondral bone in OA and RA compared to PM controls.

Conclusions: Multinucleated giant cells are associated with synovitis severity, and subchondral osteoclast numbers are increased in OA, as well as in RA. Further research targeting multinucleated giant cells is warranted to elucidate their contributions to the symptoms and joint damage associated with arthritis.

\section{Background}

Osteoarthritis (OA) is a chronic disease affecting the whole joint with progressive changes in the articular cartilage. Changes also in the synovium and subchondral bone are closely associated with symptoms [1], and are thought to contribute to cartilage damage [2]. Rheumatoid arthritis (RA) is characterized by inflammation in the synovium, and also in subchondral bone [3].

Synovitis in RA drives pain, cartilage degradation and pannus formation with subsequent erosions. It is increasingly recognized that synovitis is also observed both

\footnotetext{
* Correspondence: david.walsh@nottingham.ac.uk

${ }^{2}$ Arthritis Research UK Pain Centre, Department of Academic Rheumatology, University of Nottingham, City Hospital, Clinical Sciences Building, Hucknall Road, Nottingham NG5 1PB, UK

Full list of author information is available at the end of the article
}

in early and in late OA [4]. Indeed, synovitis predicts structural severity and progression of tibiofemoral cartilage damage in OA [5]. Histological features of OA synovitis include synovial lining hyperplasia, infiltration of macrophages and lymphocytes, angiogenesis and fibrosis [6]. OA synovitis may sometimes resemble that which is characteristic of RA. Subchondral inflammation might also contribute to increased bone turnover and joint damage in both OA and RA [7]. Although OA and RA differ in their aetiologies, with mechanical factors being key to knee OA progression and specific immunity driving RA, common mechanisms may contribute to joint damage and pain in both conditions.

Macrophage infiltration is a characteristic feature of synovitis, and is associated with radiographic joint damage both in OA [6] and in RA [8]. Macrophages can fuse 
to form multinucleated giant cells (MGCs) and this represents a cellular specialization for improved phagocytosis [9]. Although these macrophage polykaryons, generally classified into Langhans (LGC) and foreign body giant cells (FBGC), resemble osteoclasts morphologically, they differ in their functional role. FBGC derived from tissue surrounding total joint arthroplasties were found to express tartrate-resistant acid phosphatase (TRAP) [10]. In fact, many osteoclasts markers, such as TRAP, cathepsin $\mathrm{K}$ or metalloproteinase (MMP)-9, among others, can also be expressed by both FBGC and LGC at low levels [11]. In addition, the expression of specific chemokines such as chemokine ( $\mathrm{C}-\mathrm{C}$ motif) ligand (CCL) $-2,-3,-4,-5$ and -9 have been found to be highly expressed by FBGCs in comparison to osteoclasts [12]. However, whereas osteoclasts are capable of forming lacunar pits of resorption in bone, LGC and FBGC are not [13].

The formation of giant cells in non-skeletal tissues can arise as a result of chronic inflammation due to the presence of foreign material or persistent pathogens. The physiological role of MGCs includes remodelling of granuloma-associated extracellular matrix and clearance of foreign particles from tissues [14]. In vitro studies of human tuberculous granulomas have shown a phagocytic activity of LGCs in the presence of low virulence mycobacterium whereas these cells were incapable of phagocytosis in the presence of highly virulent mycobacteria, despite still retaining a strong antigen presentation capability [15]. FBGC together with their macrophage precursors adhere to different synthetic surfaces and degrade or resorb particles that are too large to permit macrophage phagocytosis [16]. Another MGC subtype, foam-like (sometimes called Touton cells), is characterized by lipid uptake [17].

MGCs have been described in inflammatory infiltrates in RA [18], but have also been noticed in the synovium of other arthritic conditions, such as tuberculous arthritis, traumatic arthritis, villonodular synovitis [19], ankylosing spondylitis, psoriasis and OA [20]. Despite the first description of synovial giant cells in RA by Grimley and Sokoloff in 1966 [21], their role in the pathogenesis of OA remains largely unknown. Some recent reports described the presence of these cells in OA synovium $[22,23]$, whereas others report few or none $[24,25]$.

Subchondral bone turnover is increased in OA, as evidenced by bone formation and resorption biomarkers [26], as well as by imaging techniques, including radiography [27], computerized tomography, magnetic resonance imaging [28], dual X-ray absorptiometry and scintigraphy [29]. Indeed, recent data from animal models suggest that subchondral bone remodelling might precede and mediate cartilage damage in OA [30], and treatments that target osteoclasts might reduce $\mathrm{OA}$ pain [31].
Although activated osteoclasts might be involved in the pathogenesis of OA and RA [32, 33], their relative abundance in the subchondral bone of these diseases remains unknown.

In this study we aimed to study the presence of MGCs in both synovium and subchondral bone tissues of patients with $\mathrm{OA}$ and further characterize their phenotypes. We also investigated their relationship with synovial inflammation, and compared our findings with MGCs seen in RA.

\section{Methods \\ Patients}

An age and sex matched cross-sectional comparative study was performed on knee samples from people with RA $(n=21)$ or OA $(n=42)$ purposively selected from the Arthritis Research UK Pain Centre Joint Tissue Repository. Informed consent was gained from each donor (for PM cases, next of kin) according to protocols approved by the United Kingdom National Research Ethics Service (Nottingham Research Ethics Committee $1(05 / \mathrm{Q} 2403 / 24)$ and Derby Research Ethics Committee $1(11 / \mathrm{H} 0405 / 2))$.

All participants in the $\mathrm{OA}$ and RA groups satisfied ACR classification criteria for knee OA [34] or RA [35]. RA samples were from consecutive cases collected at joint replacement surgery. Patients with RA were clinically examined prior to joint replacement surgery, a 28-joint Disease Activity Score (DAS28) calculated, and disease activity was classified as high, moderate or low according to European League Against Rheumatism criteria [36]. In order to explore possible associations with synovitis, OA samples, also collected at joint replacement surgery, were purposively selected according to brief histological grading of the severity of synovitis, as previously described [37], and allocated into 2 groups; non-inflamed OA (OANI; $n=21$ ) without lining hyperplasia or increased cellularity (grade 0 and 1 ), or inflamed OA (OAI, $n=21)$ with lining cell hyperplasia and marked hypercellularity (grade 3 ). The latter group represented $28 \%$ from the screened OA total knee replacement samples. In order to compare MGC phenotypic characteristics between OA and RA, and for correlation analyses, OANI and OAI samples were combined to give single OA group. Non-arthritic post-mortem (PM) cases were used as controls $(n=21)$. PM cases were selected as non-arthritic if there was no reported arthropathy on case note review, and relatives were unaware of the deceased reporting knee pain during the past year, and no Heberden's nodes or knee joint osteophytes were observed on macroscopic examination post mortem. OA and PM cases were age matched to RA cases by screening consecutive eligible cases for each disease group aged within 2 years of each index RA case. 
Synovium and mid-coronal fragments of medial tibial plateaux were collected from selected patients and used for enzymatic histochemistry and immunohistochemistry. Samples were obtained, formalin-fixed, decalcified in $10 \%$ ethylenediaminetetraacetic acid (tibial plateaux only) and paraffin embedded at Sherwood Forest Hospitals NHS Foundation Trust, Sutton in Ashfield, UK.

\section{Histopathology}

All histological assessments were undertaken by a single researcher (IP-P) who was blinded to diagnostic group. Synovitis severity was firstly graded according to Haywood et al., 2003, as criteria for inclusion of patients in both OANI and OAI groups. This score, mainly based in lining hyperplasia grading, was complemented by using the Krenn score which provides more detailed characterization of synovial inflammation using haematoxylin and eosin stained sections, as previously described in both OA and RA [38]. Briefly, in addition to lining hyperplasia, fibrovascular alterations in the interstitium and cellular infiltration were evaluated using 0-3 point subscales, where 0 indicates absence, 1 mild, 2 intermediate and 3 strong. The total score was obtained from the sum of partial grades with a maximum total score of nine. Spearman's rank correlation coefficients were used to explore association between the two methods for scoring synovitis in OA.

Synovial MGCs were identified as large cells showing three or more nuclei inside their cytoplasm in haematoxylin and eosin stained sections, thereby avoiding confounding by mitotic cells with two nuclei. Classification of different morphological variants was performed according to the arrangement and composition of their organelles. Foreign body giant cells were identified as MGCs with many nuclei diffusely distributed throughout the cytoplasm [16]. Langhans giant cells, in contrast, were recognized by the multiple nuclei arranged in a horseshoe shape [9]. Multinucleated foam giant cells were characterized by numerous nuclei clustered together, surrounded by a foamy cytoplasm [17]. Although multinucleated plasma cells are not derived from macrophage lineage, we also assessed these subtype of MGCs by their eccentric nuclei with a dense distribution of chromatin [39]. Osteoclasts were sought in synovium as TRAP-positive MGCs, and also identified in subchondral bone as TRAP-positive MGCs adjacent to bone or calcified cartilage.

Sections were analyzed through $10 \times$ and $40 \times$ objective lenses to identify MGC location within the lining and sublining layers and to assess cell morphology, respectively. An image of each MGC was captured using a 40x objective (AxioCam, Carl Zeiss Ltd., North Ryde, NSW, Australia) and maximum diameter $(\mu \mathrm{m})$ was measured with AxioVision Rel 4.8 software (Carl Zeiss Ltd.,
Welwyn Garden City, UK). Sections of synovium were scanned with EPSON Expression XL 10000 scanner (Epson America Inc, Long beach, CA, USA) for measuring the synovial biopsy area with AxioVision Rel 4.8 software (Carl Zeiss Ltd., Welwyn Garden City, UK). MGC densities are expressed as number per $\mathrm{mm}^{2}$.

TRAP enzymatic activity assay and immunohistochemistry Synovial and tibial plateaux samples were serially sectioned at $5 \mu \mathrm{m}$, dewaxed, rehydrated and incubated overnight in $1 \mathrm{mM} \mathrm{CaCl} 2$ and $1 \mathrm{mM} \mathrm{MgCl}_{2}$, then stained for TRAP, according to manufacturer's protocol (Sigma, St Louis, Mo, USA). Mid-coronal fragments of medial tibial plateaux were divided into three equal parts (external, central and internal) and TRAP positive cells with multiple nuclei were counted in each part as positive MGCs under light Axioskop-50 microscope (Carl Zeiss Ltd., Welwyn Garden City, UK), using a 40× objective lens. Total osteoclastic activity was expressed as the sum of TRAP positive cells of each part per $\mathrm{mm}^{2}$.

Macrophages were visualized using mouse monoclonal anti-CD68 primary antibody, $1 \mathrm{mg} / \mathrm{ml}$ at a $1 / 200$ dilution (Dako, clone PG-M1, M0876, Golstrup, Denmark) and a nickel-enhanced $\mathrm{ABC}$-peroxidase technique [40] (Vector laboratories, CA, USA). Cathepsin K positive cells were visualized with a mouse monoclonal anticathepsin K (Abcam, ab66237, Cambridge, UK) antibody, $0,5 \mathrm{mg} / \mathrm{ml}$ at a $1 / 100$ dilution, and $\mathrm{ABC}$-peroxidase developed with diaminobenzidine. Horse anti-mouse IgG secondary antibodies, $0,5 \mathrm{mg} / \mathrm{ml}$ (Vector laboratories, BA2001, CA, USA), were used at dilutions $1 / 100$ for CD68 and 1/4000 for cathepsin K. Antigen retrievals used were $1 \mathrm{mg} / \mathrm{ml}$ pepsin in $0.5 \mathrm{M}$ acetic acid, $2 \mathrm{~h}$ at $37^{\circ} \mathrm{C}$ for CD68, and $10 \mathrm{mM}$ citrate buffer, $\mathrm{pH} 6,30 \mathrm{~min}$ at $85^{\circ} \mathrm{C}$ for cathepsin K. Sections were counterstained with eosin for CD68 and with haematoxylin for cathepsin K; and mounted with di-n-butylphthalate in xylene (DPX mounting medium, VWR International Ltd., Lutterworth, UK).

\section{Statistical analysis}

All statistical analyses were performed using SPSS version 17.0 software for Windows (IBM, New York, NY, USA). Descriptive data are expressed as the median \pm interquartile range (IQR). Proportions were compared using Chisquared test. Associations are expressed using Spearman's rho correlation coefficient. Comparisons between multiple groups used Kruskal-Wallis tests with Bonferroni correction of post hoc Mann-Whitney U tests. $P<0.05$ was considered significant.

\section{Results}

\section{Demographic and clinical details}

Table 1 shows the demographic and clinical characteristics of participants. Age matching was successful, and 
Table 1 Demographic and clinical details

\begin{tabular}{lllll}
\hline & PM & OANI & OAl & RA \\
\hline N & 21 & 21 & 21 & 21 \\
Age, years & $68(63-73)$ & $68(63-72)$ & $68(63-72)$ & $68(63-72)$ \\
Gender, female, \% & 43 & 48 & 38 & 50 \\
ESR, mm/hr & Not available & $7(5-13)$ & $14(7-22)$ & $22(13-34)^{*}$ \\
Serum CRP, mg/L & Not available & $5(2-5)$ & $5(5-6)$ & $12(5-47)^{* \#}$ \\
DAS28 & - & - & - & $3,5(2,4-4,8)$
\end{tabular}

Data were available for erythrocyte sedimentation rate (ESR) on 13 non-inflamed OA (OANI), 18 inflamed OA (OAI) and 20 rheumatoid arthritis (RA) cases; and for serum C-reactive protein (CRP) on 12 OANI, 18 OAl and 21 RA cases. DAS28; 28 joint Disease Activity Score. Values represent medians and IQR. ${ }^{*} p<0.01$ vs. OANI; ${ }^{\#} p<0.01$ vs. OAI

there were no significant gender differences between groups. Glucocorticoids had been received by six participants (3 PM and 3 RA), and bisphosphonates by 2 RA, one also receiving calcium and vitamin $\mathrm{D}$ supplementation. Non-steroidal anti-inflammatory drugs were used in analgesic doses by 16 participants (6 OANI, 4 OAI and 6 RA). Disease modifying anti-rheumatic drugs (DMARDs) were used by 17 participants with RA (11 patients were using methotrexate, 5 sulphasalazine and 1 ciclosporin A). Five patients used a combination of 2
DMARDs, including hydroxychloroquine, rituximab or etanercept). DAS28 score showed five patients with high disease activity, eight with moderate and eight with low activity.

\section{Characterization of synovial MGC in OA and RA}

The percentage of patients who displayed MGCs in synovia was higher in RA and OAI when compared with OANI and PM $(p<0.01$, Fig. 1a). MGCs were observed in more OAI than OANI synovia, $p=0.006$, both in lining and sublining synovial layers. Absolute MGC number adjusted for synovial section area (median (IQR)) was significantly lower in OA $(0(0-0.06)$ per $\left.\mathrm{mm}^{2}\right)$ than in RA $\left(0.10(0.01-0.22)\right.$ per $\left.\mathrm{mm}^{2}, p=0.001\right)$. In OA synovia, MGCs were smaller than those observed in RA (Fig. 1b). The full range of different MGC subtypes identified by their nuclear organization was observed in each disease (Fig. 2). However, whereas Langhans-like MGCs were the most abundant subtype in OA (Fig. 1c), foreign body-like, in addition to Langhans-like MGCs, were prominent in RA synovia (Fig. 1d). Foam-like and plasma-like MGCs were less abundant in both disease groups. Foam-like MGCs were identified near to and surrounding fat cells in inflamed synovia from patients with either OA or RA. Furthermore,

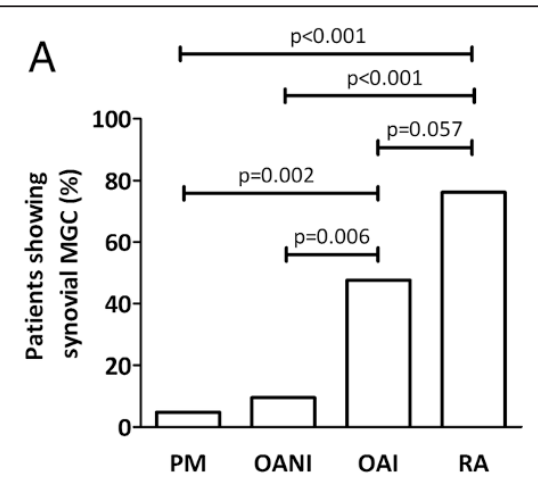

B
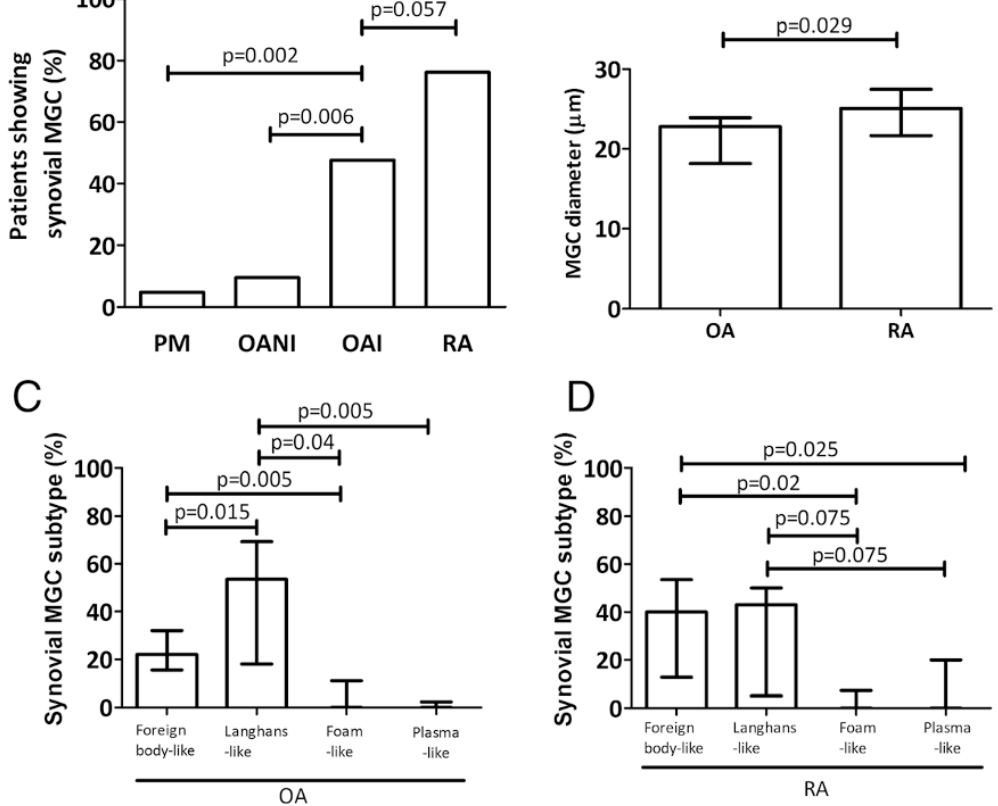

Fig. 1 Characterization of MGC subtypes in OA and in RA. a. A greater proportion of inflamed OA (OAI) and RA cases displayed MGCs than did non-inflamed OA (OANI) or non-arthritic (post mortem, PM) controls. b. Increased MGC size in RA compared with OA cases. $\mathbf{c}$ and $\mathbf{d}$. Relative abundance of morphological MGC subtypes observed in OA (c) and RA (d) synovia. Bars in b-d represent medians, with IQRs indicating between MGC heterogeneity 


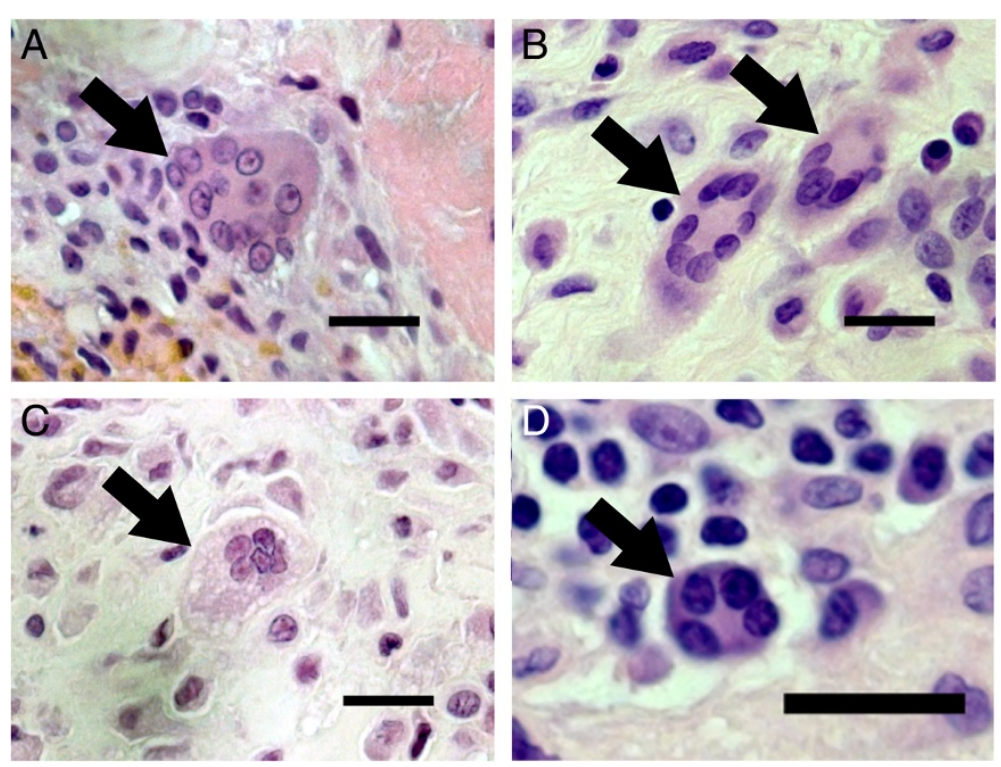

Fig. 2 Morphological characterization of synovial MGC subtypes in OA and in RA. Foreign body giant cells were identified as MGCs with many nuclei diffusely distributed throughout the cytoplasm (a). Langhans giant cells were recognized by the multiple nuclei arranged in a horseshoe shape (b). Foam MGC subtype was characterized by numerous nuclei clustered together, surrounded by a foamy cytoplasm (c). Plasma MGC subtype was assessed by their eccentric nuclei with a dense distribution of chromatin (d). Overall MGC subtypes were identified in either OA or RA. Arrows indicate each different MGC subtype in synovium. Haematoxylin and eosin staining. Scale bars $=20 \mu m$

multiple mononuclear CD68 positive cells were found in a crown-like structure encircling adipocyte cells (Additional file 1).

We also analyzed TRAP activity and Cathepsin $\mathrm{K}$ immunoreactivity as markers of MGCs in synovium [41]. We found that most of the foreign body-like MGCs positively stained for TRAP, whereas many MGCs displaying characteristics of Langhans, multinucleated foam-like and plasma cells were TRAP negative (Figs. 3a and $\mathrm{b})$. Cathepsin K-immunoreactive MGCs were only localized to synovial pannus adjacent to bone surfaces in RA tibial plateau (Fig. 3c), and were not observed in synovial tissue distant from bone or cartilage. Some mononuclear synovial lining and sublining cells also stained positive for cathepsin K, both in OAI and in RA synovia (Figs. 3d and e).

\section{Synovial inflammation in OA and RA}

Haywood synovitis score was significantly correlated with Krenn score in patients with OA $(r=0791 ; p<0001)$. OAI and RA samples were each characterized by synovial lining hyperplasia, sublining fibrosis and stromal vascularization, along with inflammatory infiltrates often containing follicle-like lymphoid aggregates (Figs. 4a-d). OAI and RA synovia had higher synovitis grades than OANI and PM (each $p<0.001$, Fig. 4e). Scores for each Krenn subscale (stromal cell density, lining hyperplasia and inflammatory infiltrate) were increased in OAI and RA synovia in comparison with OANI and PM (each $p<0.05)$.
Synovitis grade and synovial MGC density were significantly associated, both in OA and RA groups (Fig. 4f). Furthermore, MGC densities were higher in cases with greater inflammatory infiltration in OA $(r=0.63 ; p=0.03)$, and with higher stromal cellularity in RA $(r=0.53 ; p=$ 0.03). Associations between serum CRP levels and MGC density did not reach statistical significance in either OA or RA $(r=0.30 ; p=0.38$ and $r=0.09 ; p=0.75$ respectively). Likewise, serum ESR was not significantly associated with MGC density in OA or RA $(r=-0.20 ; p=0.55$ and $r=-0.12 ; p=0.68$, respectively).

\section{Subchondral bone osteoclasts in OA and RA}

We then analyzed the presence of multinucleated cells in subchondral bone of medial tibial plateaux. MGCs adjacent to bone surfaces that displayed TRAP activity and a random nuclear organization were counted as osteoclasts. Increased numbers of TRAP-positive osteoclasts were observed in subchondral bone from people with RA, or with $\mathrm{OA}$ and either non inflamed or inflamed synovium (Figs. 5b-d) compared with non-arthritic PM controls, $P<$ 0.05 in all comparisons (Fig. 5a). No significant differences in osteoclast numbers were found between OAI and RA groups, whereas TRAP-positive subchondral osteoclasts were more abundant in RA compared to the noninflamed OA group, $p=0.036$ (Fig. 5e). Associations between subchondral TRAP-positive osteoclasts and synovial MGC numbers were not statistically significant in either OA $(r=0.30, P=0.62)$ or RA $(r=-0.14 ; P=0.76)$. 

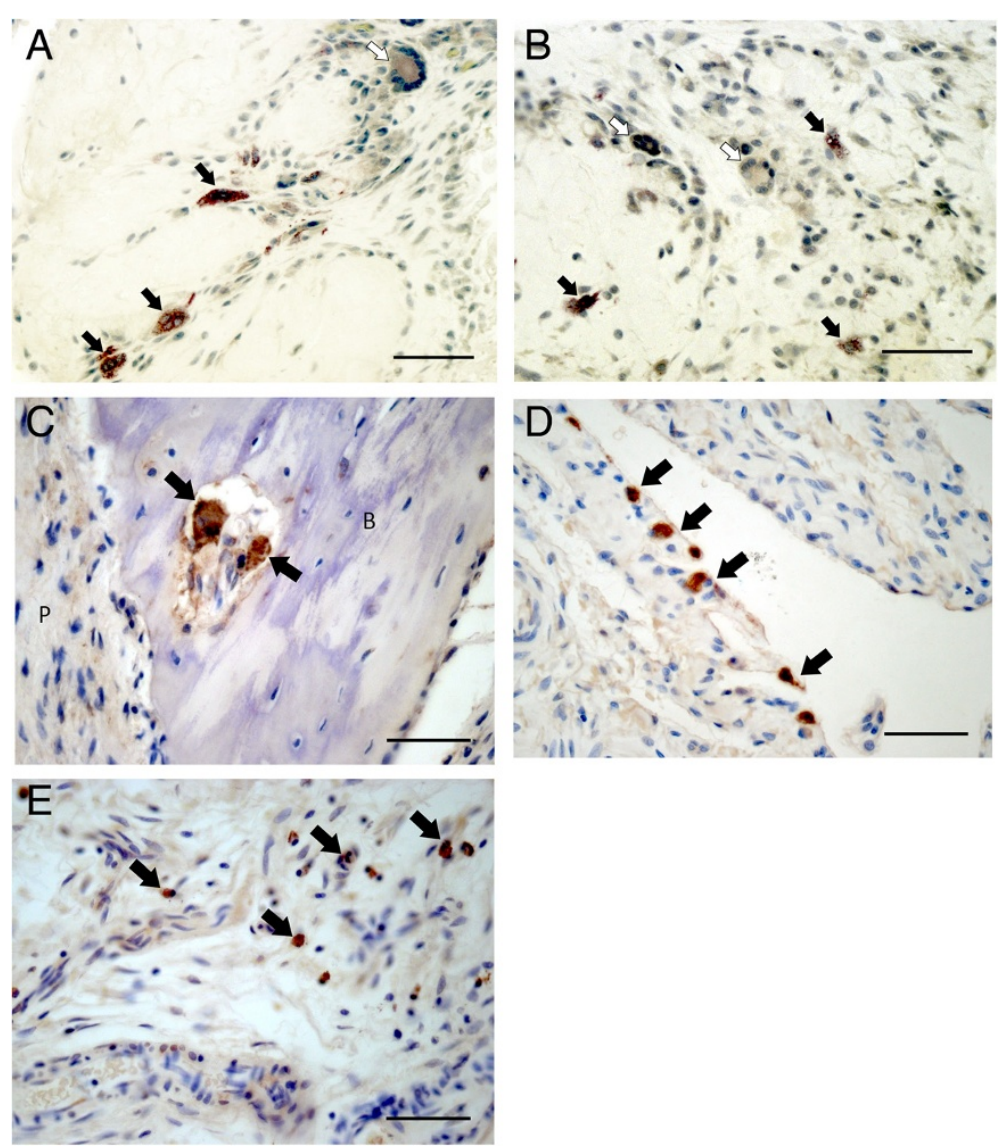

Fig. 3 TRAP and cathepsin $\mathrm{K}$ enzyme activity in synovium from OA and RA patients. $\mathbf{a}$ and $\mathbf{b}$. Representative synovial TRAP-positive and TRAP-negative MGCs both in inflamed OAI (a) and RA (b) cases. c. Cathepsin K-immunoreactive MGC localized to synovial pannus adjacent to bone surface in RA and OA tibial plateaux. $\mathbf{d}$ and $\mathbf{e}$. Cathepsin K-immunoreactive mononuclear synovial lining (d) and sublining (e) cells in OAl and in RA synovium. Filled arrows indicate TRAP-positive or cathepsin K-positive cells. Open arrows indicate TRAP-negative MGCs. $P=$ pannus and $B=$ bone, scale bars $=50 \mu m$

\section{Discussion}

In this study we have shown that MGCs were increased in OA, especially in inflamed synovia which presented similar MGC numbers to those observed in RA. MGC subtypes might differ between OA and RA, with a relative sparsity of foreign body MGCs in OA. In addition, we provide histopathological evidence of increased subchondral osteoclastic activity in OA, as well as in RA. Together, our findings indicate a clear activation of mononuclear phagocyte system cells, both in synovium and in subchondral bone, and both in OA and in RA.

Synovial inflammation is a common feature of OA, although the intensity and nature of inflammation may differ between OA and RA [42]. It is currently unknown whether synovitis characterizes a subgroup of people with OA, or may occur during phases of the disease in all patients. In cross sectional studies, the extent of synovitis varies between cases, ranging from normal to severe inflammation [43]. Cases purposively selected for the current study represented both ends of this spectrum in order to explore associations between
MGCs and synovitis, and to characterize MGCs in detail. The inflamed OA group studied here, comprised $28 \%$ of consecutive cases donating tissue at joint replacement surgery for $\mathrm{OA}$, and therefore represents a common inflammatory phenotype amongst people with end stage OA. A similar prevalence of synovitis was observed in a previous study of 104 participants undergoing joint replacement surgery for knee OA [37].

We have also demonstrated that there is a prevalent subgroup of people with OA in whom the numbers of MGCs are comparable to those in people with RA at the time of joint replacement surgery. Furthermore, MGC densities were significantly associated with the total synovitis score indicating that this is a cellular characteristic of synovitis that is shared between OA and RA, suggesting shared inflammatory mechanisms. Varying estimates of the prevalence of MGCs in synovium from people with OA or RA might reflect the degree of synovitis in cases selected for study.

In the current study, statistically significant associations were not detected between ESR or serum CRP and 

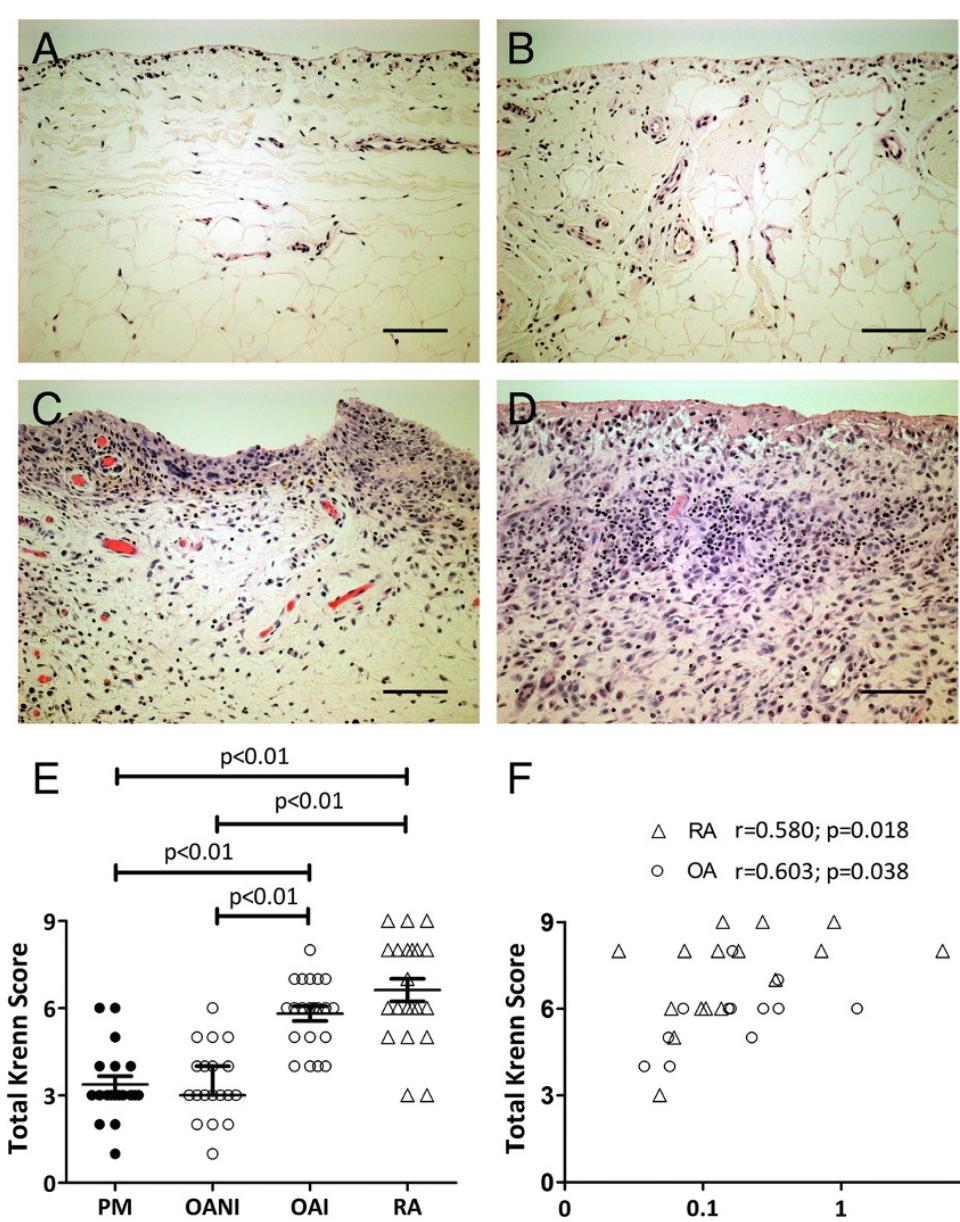

$\mathrm{F}$

$\triangle$ RA $r=0.580 ; p=0.018$

○ $O A \quad r=0.603 ; p=0.038$

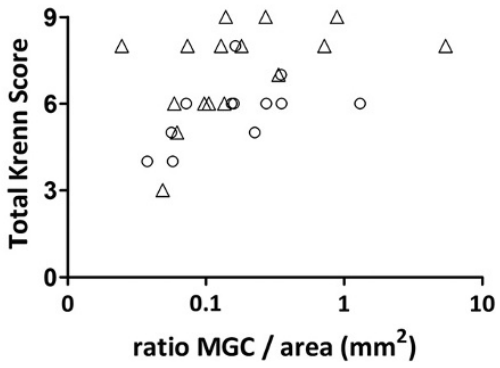

Fig. 4 Synovial inflammation in OA and RA. a-d. Representative sections of synovia from cases with median Krenn scores for each disease group stained with haematoxylin and eosin. Panels represent post-mortem, PM (a), non-inflamed OA, OANI (b), inflamed OA, OAI (c) and RA (d) cases. Scale bars $=100 \mu \mathrm{m}$. e. Synovitis, determined as total Krenn score was greatest in OAl and RA cases. $\mathbf{f}$. Numbers of MGCs per area of synovium were positively correlated with synovitis scores both in OA and in RA cases

MGC numbers, suggesting local co-ordination of MGCs and inflammation within the synovium. However, larger populations would need to be investigated to explore smaller possible systemic influences on MGC formation. A limitation of cross sectional studies such as ours is that differences between groups may be caused by unmeasured confounding factors. We have attempted to minimize possible confounding by carefully matching cases by gender and age and although our assessments were undertaken blinded to diagnostic group, we cannot be certain that detection of differences between groups was entirely free from observer bias. Participants with RA were more likely to be using glucocorticoids, bisphosphonates or DMARDs than were those with OA, and inhibition of MGCs by anti-inflammatory drugs might have led us to underestimate the magnitude of increased MGC and osteoclast numbers in RA.
Our data extend those from a previous study showing two main types of giant cells in arthritic synovium [44]. We have demonstrated that OA and RA synovia exhibit distinct patterns of MGC subtype frequency, Langhanslike MGC being the predominant subtype in people with OA. Langhans-like MGCs contribute to processing and presentation of antigens and the development of specific immune responses, and their presence in OA might indicate a contribution of specific immunity to OA [45-47]. The pathological relevance of smaller MGC size and lower prevalence of foreign body like giant cells in OA than in RA remains unclear, but we speculate that deficiencies in processing cartilage degradation products might contribute to synovitis in OA.

In addition to the main Langhans and foreign-body MGC subtypes, we also observed foam-like MGCs in synovia from people undergoing joint replacement 


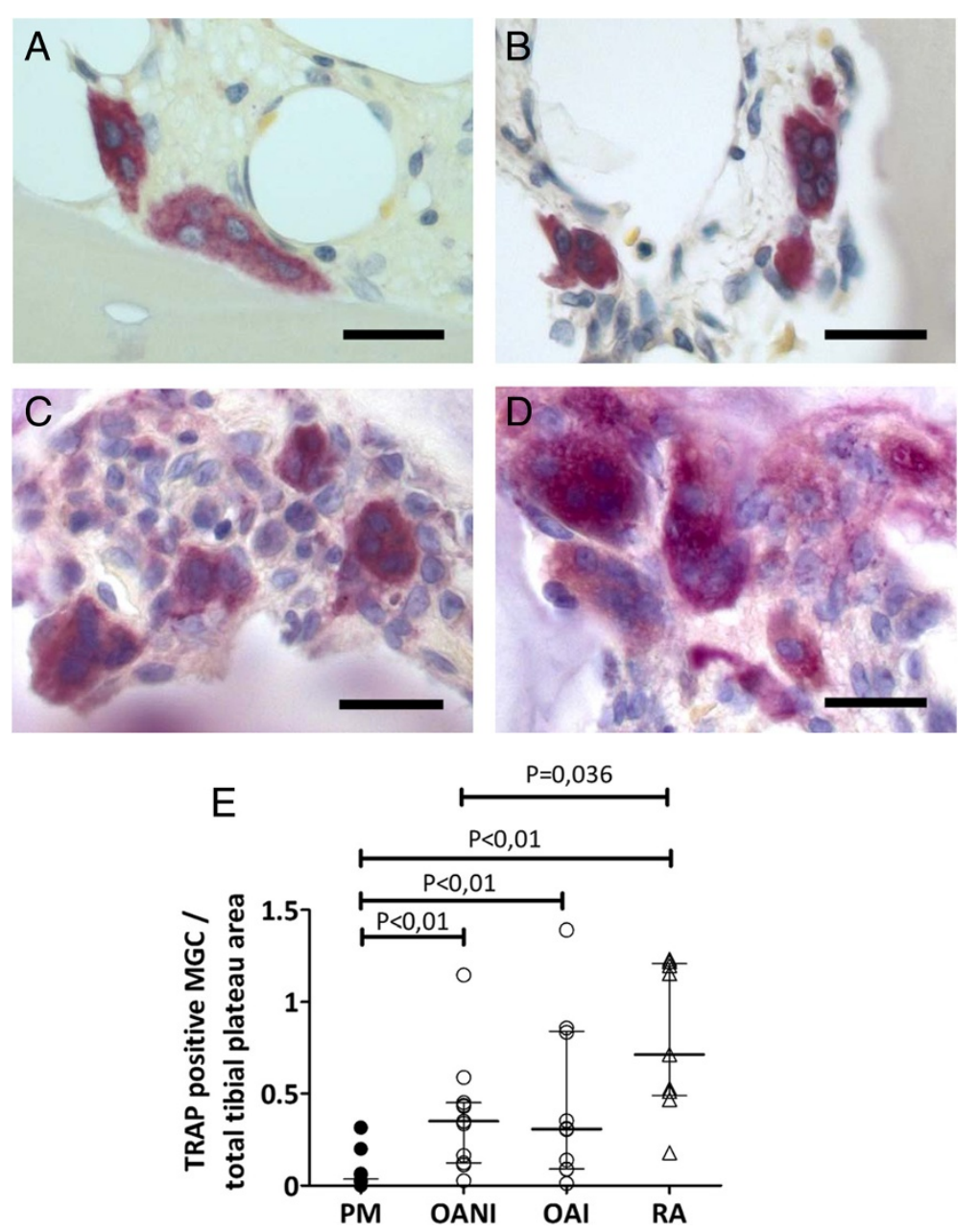

Fig. 5 Subchondral bone TRAP positive osteoclast in OA and in RA. a-d. Representative sections from non-arthritic patients (a), with OANI (b), OAI (c) and RA (d) showing TRAP positive osteoclasts adjacent to bone surfaces. Scale bars $=20 \mu \mathrm{m}$. e. c. Absolute TRAP-positive osteoclast number adjusted for the area of mid-coronal tibial plateau fragments. Bars represent medians with IQRs

surgery for either OA or RA. Previous studies have most frequently localized foam-like MGCs in lesions containing cholesterol and lipid deposits, such as xanthomas or xanthogranulomas [48], and these MGCs might develop when a cell fusion stimulus is also accompanied by a factor stimulating lipid uptake [49]. The contribution of foam-like MGCs to synovitis in OA and RA deserves further study, given recognized associations between human arthritis, obesity and metabolic syndrome [50], and preclinical evidence that synovial foam-like MGCs might be associated with increased bone resorption [51].

We found that foreign body-like MGCs positively stained for TRAP, indicating that these cells might have osteoclastic activity [52], whereas Langhans MGCs were negative for TRAP. Absence of TRAP activity in Langhans MGCs has also been noted in canine and feline neoplasia [53]. Cathepsin $\mathrm{K}$ was not a specific marker of MGC in inflamed synovium, where it was also localized to mononuclear cells more distant from bone and cartilage. We did not identify cathepsin $\mathrm{K}$ activity in MGCs that were distant from bone or cartilage in human synovia, in contrast to previous suggestions that cathepsin K-positive staining might be a general feature of MGCs [41]. Cathepsin K-negative MGCs have also been reported in human breast carcinoma and lung biopsies [54]. Our findings suggest that cathepsin K-immunoreative MGCs in human synovium might represent an osteoclastic MGC subtype. However, we cannot be certain whether TRAP and cathepsin $\mathrm{K}$ positive MGCs detected in the synovium were osteoclasts, or alternatively osteoclast precursors. Diverse MGC subtypes have been described previously in various autoimmune, infectious or sarcoid granulomatous diseases. The demonstration of diverse morphological subtypes [14], distribution and immunohistochemical characteristics [55] might reflect discrete functional states of MGCs.

TRAP and cathepsin K positive osteoclasts were localised adjacent to bone and cartilage, both in synovium 
and in subchondral bone. The formation of resorption subchondral pits by osteoclasts [56] and cells that resorb mineralized and unmineralized articular cartilage [57] contribute to hard tissue remodeling in OA. Increased subchondral bone turnover and resorption might contribute to OA development, and might be features of both early and end stage disease [58]. Our findings suggest a contribution of increased osteoclast activation to increased subchondral bone remodelling both in RA and in OA. Increased numbers of TRAP-positive MGCs in OA subchondral bone reflect augmented osteoclastic activity in animal models of OA [59], and our findings suggest similarly increased subchondral osteoclastic activity in human knee OA. Future studies should explore possible associations between subchondral osteoclast numbers and biochemical or imaging markers of subchondral bone turnover. Subchondral osteoclast numbers were increased in both inflamed and noninflamed OA subgroups, whereas synovial MGCs were more frequently observed in the inflamed subgroup and were associated with synovitis grade. Furthermore, no statistically significant association was found between the increased numbers of subchondral osteoclasts and synovial MGCs. These data lead us to suggest that synovial inflammation and subchondral bone remodelling each may be regulated by different mediators.

\section{Conclusion}

We found that MGCs were increased in OA, especially in inflamed OA which presented similar MGC numbers to those observed in RA. Synovial MGCs displayed a range of morphological subtypes, with Langhans giant cells being the predominant variant in OA, and both Langhans and foreign body like subtypes in RA. MGCs might contribute to catabolic activity and be regulated by local factors within the synovium, whereas MGCs in subchondral bone might predominantly represent osteoclasts and be regulated by factors that are independent of synovitis. Preclinical studies and early clinical trials support the targeting of osteoclasts in the treatment of OA [31]. Further research is required to determine the potential of targeting MGCs to improve pain and joint damage both in OA and in RA.

\section{Additional file}

Additional file 1: Morphological MGC foam subtype and CD68 positive cells surrounding fat cells in OA and in RA. A and B. MGCs displaying a foam-like subtype were identified near to and surrounding fat cells in inflamed synovia from patients with either OA (A) or RA (B). Haematoxylin and eosin staining. Scale bar $=20 \mu \mathrm{m}$. Open arrows indicate foam-like MGC and A = adipocyte. C and D. Multiple mononuclear CD68 positive cells were found in a crown-like structure encircling adipocyte cells in both $\mathrm{OA}(\mathrm{C})$ and RA (D). Immunohistochemistry for CD68, using eosin contrast. Scale bar $=100 \mu \mathrm{m}$. (TIFF $3238 \mathrm{~kb}$ )

\section{Abbreviations}

ACR: American college of rheumatology; CRP: C reactive protein; DMARDs: Disease modifying anti-rheumatic drugs; ESR: Erythrocyte sedimentation rate; FBGC: Foreign body giant cell; IQR: Interquartile range; LGC: Langhans giant cell; MGC: Multinucleated giant cell; OA: Osteoarthritis; OAl: Inflamed osteoarthritis; OANI: Non-inflamed Osteoarthritis; PM: Post-mortem; RA: Rheumatoid arthritis.

\section{Competing interests}

The authors declare that they have no competing interests.

\section{Authors' contributions}

IP-P conceived of the study, participated in its design, acquisition of data, performed statistical analysis and interpretation of data, and drafted the manuscript. RL and JAR-B participated in the interpretation of data and helped to draft the manuscript. GH-B conceived of the study and design, and helped to draft the manuscript. DAW conceived of the study, design and coordination, interpretation of data and helped to draft the manuscript. All authors read and approved the final manuscript.

\section{Acknowledgements}

We are grateful to all the patients, the orthopaedic surgeons and the Bereavement Centre at the Sherwood Forest Hospitals NHS Trust for providing clinical material. We also thank Deborah Wilson for patient recruitment and assessment, and Roger Hill for tissue collection and processing at Sherwood Forest Hospitals NHS Foundation Trust. This work was partially supported by the Arthritis Research UK Pain Centre (Arthritis Research UK, grant number 18769) and by research grant from the Instituto Carlos III (PI13/00570 and PI12/00144). IP-P was funded by an ECTS exchange scholarship grant and an investigator mobility grant from the Autonomous University of Madrid.

\section{Author details}

'Bone and Joint Research Unit, Service of Rheumatology, IIS-Fundación Jiménez Díaz, Autonomous University of Madrid, Avda Reyes Católicos, 2, Madrid 28040, Spain. ${ }^{2}$ Arthritis Research UK Pain Centre, Department of Academic Rheumatology, University of Nottingham, City Hospital, Clinical Sciences Building, Hucknall Road, Nottingham NG5 1PB, UK.

Received: 5 May 2015 Accepted: 10 August 2015

Published online: 27 August 2015

\section{References}

1. Stoppiello LA, Mapp PI, Wilson D, Scammell BE, Orth F, Walsh DA. Structural associations of symptomatic knee osteoarthritis. Arthritis Rheum. 2014;66:3018-27.

2. Loeser RF, Goldring SR, Scanzello CR, Goldring MB. Osteoarthritis: a disease of the joint as an organ. Arthritis Rheum. 2012;64:1697-707.

3. Woolley DE, Tetlow LC. Observations on the microenvironmental nature of cartilage degradation in rheumatoid arthritis. Ann Rheum Dis. 1997:56:151-61.

4. Benito M, Veale D, FitzGerald O, Van den Berg W, Bresnihan B. Synovial tissue inflammation in early and late osteoarthritis. Ann Rheum Dis. 2005;64:1263-7.

5. Ayral X, Pickering EH, Woodworth TG, Mackillop N, Dougados M. Synovitis: a potential predictive factor of structural progression of medial tibiofemoral knee osteoarthritis - results of a 1 year longitudinal arthroscopic study in 422 patients. Osteoarthritis Cartilage. 2005:13:361-7.

6. Scanzello CR, Goldring SR. The role of synovitis in osteoarthritis pathogenesis. Bone. 2012;51:249-57.

7. Walsh DA, McWilliams DF, Turley MJ, Dixon MR, Fransès RE, Mapp PI, et al. Angiogenesis and nerve growth factor at the osteochondral junction in rheumatoid arthritis and osteoarthritis. Rheumatology (Oxford). 2010;49:1852-61

8. Mulherin D, Fitzgerald O, Bresnihan B. Synovial tissue macrophage populations and articular damage in rheumatoid arthritis. Arthritis Rheum. 1996:39:115-24.

9. Anderson JM. Multinucleated giant cells. Curr Opin Hematol. 2000;7:40-7.

10. Anderson JM, Rodriguez A, Chang DT. Foreign body reaction to biomaterials. Semin Immunol. 2008;20:86-100.

11. Cowan RW, Singh G. Giant cell tumor of bone: A basic science perspective. Bone. 2012;52:238-46. 
12. Khan UA, Hashimi SM, Khan S, Quan J, Bakr MM, Forwood MR, et al. Differential expression of chemokines, chemokine receptors and proteinases by foreign body giant cells (FBGCs) and osteoclasts. J Cell Biochem. 2014;115:1290-8.

13. Maggiani F, Forsyth R, Hogendoorn PCW, Krenacs T, Athanasou NA. The immunophenotype of osteoclasts and macrophage polykaryons. J Clin Pathol. 2011:64:701-5.

14. Quinn MT, Schepetkin IA. Role of NADPH oxidase in formation and function of multinucleated giant cells. J Innate Immun. 2009;1:509-26.

15. Lay $G$, Poquet $Y$, Puissegur $M$, Botanch $C$, Bon $H$, Levillain $F$, et al. Langhans giant cells from $M$. tuberculosis -induced human granulomas cannot mediate mycobacterial uptake. J Pathol. 2007;211:76-85.

16. Brodbeck WG, Anderson JM. Giant cell formation and function. Curr Opin Hematol. 2009;16:53-7

17. Gómez-Mateo MDC, Monteagudo C. Nonepithelial skin tumors with multinucleated giant cells. Semin Diagn Pathol. 2013;30:58-72.

18. Schett G. Cells of the synovium in rheumatoid arthritis. Osteoclasts. Arthritis Res Ther. 2007;9:203.

19. Bhan AK, Roy S. Synovial giant cells in rheumatoid arthritis and other joint diseases. Ann Rheum Dis. 1971;30:294-8.

20. Soren A, Waugh TR. The giant cells in the synovial membrane. Ann Rheum Dis. 1981;40:496-500

21. Grimley P, Sokoloff L. Synovial giant cells in rheumatoid arthritis. Am J Pathol. 1966:49:931-54

22. Ogawa K, Mawatari M, Komine M, Shigematsu M, Kitajima M, Kukita A, et al. Mature and activated osteoclasts exist in the synovium of rapidly destructive coxarthrosis. J Bone Miner Metab. 2007;25:354-60.

23. Seitz S, Zustin J, Amling M, Rüther W, Niemeier A. Massive accumulation of osteoclastic giant cells in rapid destructive hip disease. J Orthop Res. 2014:32:702-8

24. Ashton BA, Ashton IK, Marshall MJ, Butler RC. Localisation of vitronectin receptor immunoreactivity and tartrate resistant acid phosphatase activity in synovium from patients with inflammatory or degenerative arthritis. Ann Rheum Dis. 1993;52:133-7.

25. Wilkinson LS, Pitsillides AA, Edwards JC. Giant cells in arthritic synovium. Ann Rheum Dis. 1993;52:182-4.

26. Rousseau JC, Garnero P. Biological markers in osteoarthritis. Bone. 2012:51:265-77.

27. Woloszynski T, Podsiadlo P, Stachowiak GW, Kurzynski M, Lohmander LS, Englund M. Prediction of progression of radiographic knee osteoarthritis using tibial trabecular bone texture. Arthritis Rheum. 2012;64:688-95.

28. Hunter DJ. Insights from Imaging on the Epidemiology and Pathophysiology of Osteoarthritis. Radiol Clin North Am. 2009;47(4):539-51.

29. Roman-Blas JA, Castañeda S, Largo R, Lems WF, Herrero-Beaumont G. An OA phenotype may obtain major benefit from bone-acting agents. Semin Arthritis Rheum. 2014:43:421-8.

30. Bucklandwright C. Subchondral bone changes in hand and knee osteoarthritis detected by radiography. Osteoarthr Cartil. 2004;12:10-9.

31. Sagar DR, Ashraf S, Xu L, Burston JJ, Menhinick MR, Poulter CL, Bennett AJ, Walsh DA, Chapman V: Osteoprotegerin reduces the development of pain behaviour and joint pathology in a model of osteoarthritis. Ann rheum Dis 2013 [Epub ahead of print].

32. Bugatti S, Caporali R, Manzo A, Vitolo B, Pitzalis C, Montecucco C. Involvement of subchondral bone marrow in rheumatoid arthritis: lymphoid neogenesis and in situ relationship to subchondral bone marrow osteoclast recruitment. Arthritis Rheum. 2005;52:3448-59.

33. Schett G, Stolina M, Bolon B, Middleton S, Adlam M, Brown H, et al. Analysis of the kinetics of osteoclastogenesis in arthritic rats. Arthritis Rheum. 2005:52:3192-201.

34. Altman R, Asch E, Bloch D, Bole G, Borenstein D, Brandt $K$, et al. Development of criteria for the classification and reporting of osteoarthritis. Classification of osteoarthritis of the knee. Arthritis Rheum. 1986;29:1039-49.

35. Arnett F, Edworthy S, Bloch D. The american rheumatism association 1987 revised criteria for the classification of the rheumatoid arthritis. Arthritis Rheum. 1988:31:315-24.

36. Fransen J, van Riel PLCM. The Disease Activity Score and the EULAR response criteria. Clin Exp Rheumatol. 2005;23(5 Suppl 39):S93-9.

37. Haywood L, McWilliams DF, Pearson Cl, Gill SE, Ganesan A, Wilson D, et al. Inflammation and angiogenesis in osteoarthritis. Arthritis Rheum. 2003:48:2173-7.

38. Krenn V, Morawietz L, Häupl T, Neidel J, Petersen I, König A. Grading of chronic synovitis-a histopathological grading system for molecular and diagnostic pathology. Pathol Res Pract. 2002;198:317-25.
39. Perry M, Mustafa Y, Wood S, Cawley M. Binucleated and multinucleated forms of plasma cells in synovia from patients with rheumatoid arthritis. Rheumatol Int. 1997;17:169-74.

40. Shu SY, Ju G, Fan LZ. The glucose oxidase-DAB-nickel method in peroxidase histochemistry of the nervous system. Neurosci Lett. 1988;85:169-71.

41. Park JK, Rosen A, Saffitz JE, Asimaki A, Litovsky SH, Mackey-Bojack SM, et al. Expression of cathepsin $\mathrm{K}$ and tartrate-resistant acid phosphatase is not confined to osteoclasts but is a general feature of multinucleated giant cells: systematic analysis. Rheumatology (Oxford). 2013;52:1529-33.

42. Wenham CYJ, Conaghan PG. The role of synovitis in osteoarthritis. Ther Adv Musculoskelet Dis. 2010;2:349-59.

43. Walsh DA, Yousef A, McWilliams DF, Hill R, Hargin E, Wilson D. Evaluation of a Photographic Chondropathy Score (PCS) for pathological samples in a study of inflammation in tibiofemoral osteoarthritis. Osteoarthritis Cartilage. 2009:17:304-12.

44. Koizumi F, Matsuno H, Wakaki K, Ishii Y, Kurashige $Y$, Nakamura H. Synovitis in rheumatoid arthritis: scoring of characteristic histopathological features. Pathol Int. 1999;49:298-304.

45. Scanzello CR, Plaas A, Crow MK. Innate immune system activation in osteoarthritis: is osteoarthritis a chronic wound? Curr Opin Rheumatol. 2008;20(5):565-72.

46. Gómez R, Villalvilla A, Largo R, Gualillo O, Herrero-Beaumont G: TLR4 signalling in osteoarthritis-finding targets for candidate DMOADs. Nat Rev Rheumatol 2015;11(3):159-70.

47. De Lange-Brokaar BJE, loan-Facsinay A, van Osch GJVM, Zuurmond A, Schoones J, Toes REM, et al. Synovial inflammation, immune cells and their cytokines in osteoarthritis: a review. Osteoarthritis Cartilage. 2012;20:1484-99.

48. Consolaro A, Sant'Ana E, Lawall MA, Consolaro MFMO, Bacchi CE. Gingival juvenile xanthogranuloma in an adult patient: case report with immunohistochemical analysis and literature review. Oral Surg Oral Med Oral Pathol Oral Radiol Endod. 2009;107:246-52.

49. Aterman K, Remmele W, Smith M. Karl Touton and His "Xanthelasmatic Giant Cell" A Selective Review of Multinucleated Giants Cells. Am J Dermatopathol. 1988;10:257-69.

50. Ferraz-Amaro I, González-Juanatey C, López-Mejias R, Riancho-Zarrabeitia L, González-Gay MA. Metabolic syndrome in rheumatoid arthritis. Mediators Inflamm. 2013;2013:11.

51. Prieto-Potin I, Roman-Blas JA, Martinez-Calatrava MJ, Gomez R, Largo R, Herrero-Beaumont G. Hypercholesterolemia boosts joint destruction in chronic arthritis. An experimental model aggravated by foam macrophage infiltration. Arthritis Res Ther. 2013;15:R81.

52. Connor JR, Dodds RA, James IE, Gowen M. Human osteoclast and giant cell differentiation: the apparent switch from nonspecific esterase to tartrate resistant acid phosphatase activity coincides with the in situ expression of osteopontin mRNA. J Histochem Cytochem. 1995;43:1193-201.

53. Jösten $M$, Rudolph $R$. Methods for the differentiation of giant cells in canine and feline neoplasias in paraffin sections. Zentralbl Vet A. 1997;44:630.

54. Park JK, Askin F, Giles JT, Halushka MK, Rosen A, Levine SM. Increased generation of TRAP expressing multinucleated giant cells in patients with granulomatosis with polyangiitis. PLoS One. 2012;7:e42659.

55. Nordborg E, Bengtsson B, Petursdottir V, Nordborg C. Morphological aspects of giant cells in giant cell arteritis: an electron-microscopic and immunocytochemical study. Clin Exp Rheumatol. 1997;15:129-34.

56. Shibakawa A, Yudoh K, Masuko-Hongo K, Kato T, Nishioka K, Nakamura H. The role of subchondral bone resorption pits in osteoarthritis: MMP production by cells derived from bone marrow. Osteoarthr Cartil. 2005;13:679-87.

57. Knowles HJ, Moskovsky L, Thompson MS, Grunhen J, Cheng X, Kashima TG, et al. Chondroclasts are mature osteoclasts which are capable of cartilage matrix resorption. Virchows Arch. 2012;461:205-10.

58. Karsdal MA, Bay-Jensen AC, Lories RJ, Abramson S, Spector T, Pastoureau P, et al. The coupling of bone and cartilage turnover in osteoarthritis: opportunities for bone antiresorptives and anabolics as potential treatments? Ann Rheum Dis. 2014;73:336-48.

59. Botter SM, van Osch GJVM, Clockaerts S, Waarsing JH, Weinans $H$, van Leeuwen JPTM. Osteoarthritis induction leads to early and temporal subchondral plate porosity in the tibial plateau of mice: An in vivo micro CT study. Arthritis Rheum. 2011;63:2690-9. 